\title{
Impaired Transmission in the Corticospinal Tract and Gait Disability in Spinal Cord Injured Persons
}

\author{
Dorothy Barthélemy, ${ }^{1,6}$ Maria Willerslev-Olsen, ${ }^{1}$ Henrik Lundell, ${ }^{1,3}$ Bernard A. Conway, ${ }^{5}$ Hanne Knudsen, ${ }^{4}$ \\ Fin Biering-Sørensen, ${ }^{2,4}$ and Jens Bo Nielsen ${ }^{1}$ \\ ${ }^{1}$ Department of Exercise and Sport Sciences and Department of Neuroscience and Pharmacology; ${ }^{2}$ Faculty of Health Sciences, University \\ of Copenhagen, Copenhagen; ${ }^{3}$ Danish Research Centre for Magnetic Resonance, Copenhagen University Hospital, Hvidovre; ${ }^{4}$ Clinic for \\ Spinal Cord Injuries, Rigshospitalet, Hornbak, Denmark; ${ }^{5}$ Bioengineering Unit, University of Strathclyde, Glasgow, United Kingdom; \\ and ${ }^{6}$ School of Rehabilitation, Université de Montréal, Montreal, Canada
}

Submitted 26 April 2010; accepted in final form 14 June 2010

\begin{abstract}
Barthélemy D, Willerslev-Olsen M, Lundell H, Conway BA, Knudsen H, Biering-Sørensen F, Nielsen JB. Impaired transmission in the corticospinal tract and gait disability in spinal cord injured persons. J Neurophysiol 104: 1167-1176, 2010. First published June 16, 2010; doi:10.1152/jn.00382.2010. Rehabilitation following spinal cord injury is likely to depend on recovery of corticospinal systems. Here we investigate whether transmission in the corticospinal tract may explain foot drop (inability to dorsiflex ankle) in persons with spinal cord lesion. The study was performed in 24 persons with incomplete spinal cord lesion $(\mathrm{C} 1$ to $\mathrm{L} 1)$ and 15 healthy controls. Coherence in the $10-$ to $20-\mathrm{Hz}$ frequency band between paired tibialis anterior muscle (TA) electromyographic recordings obtained in the swing phase of walking, which was taken as a measure of motor unit synchronization. It was significantly correlated with the degree of foot drop, as measured by toe elevation and ankle angle excursion in the first part of swing. Transcranial magnetic stimulation was used to elicit motor-evoked potentials (MEPs) in the TA. The amplitude of the MEPs at rest and their latency during contraction were correlated to the degree of foot drop. Spinal cord injured participants who exhibited a large foot drop had little or no MEP at rest in the TA muscle and had little or no coherence in the same muscle during walking. Gait speed was correlated to foot drop, and was the lowest in participants with no MEP at rest. The data confirm that transmission in the corticospinal tract is of importance for lifting the foot during the swing phase of human gait.
\end{abstract}

\section{N T R O D U C T I O N}

It has been demonstrated in recent years that it is possible to drive neuroplastic changes in the brain and spinal cord and thereby promote some recovery of lost function through appropriately designed interventions (Hummel and Cohen 2005; Nudo 2006; Plow et al. 2009). Development of such interventions will depend on gaining a detailed understanding of the underlying physiological mechanisms that support recovery and on our ability to chart over time the plastic changes that are induced as a consequence of an intervention (Ellaway et al. 2004). With recent advances in imaging and electrophysiological techniques we are beginning to understand the mechanisms responsible for reorganizing the central control of movement at spinal and supraspinal levels following injury and/or therapy. However, this still constitutes a challenge, especially for the evaluation of gait and the mechanisms involved in its control.

Address for reprint requests and other correspondence: J. B. Nielsen, Department of Neuroscience and Pharmacology, Department of Physical Exercise and Sport Sciences, University of Copenhagen, Panum Institute 18.5, Blegdamsvej 3, 2200 Copenhagen N, Denmark (E-mail: jbnielsen@sund.ku.dk).
Indeed, gait involves not only a complex interaction of many muscles and limb segments, but also an interaction of spinal neuronal networks, sensory feedback mechanisms, and supraspinal commands (Hultborn and Nielsen 2007; Nielsen 2003; Rossignol 2006).

Animal experiments have shown that the motor cortex is mainly of importance for stepping over obstacles and for motivational and visual adjustments of gait pattern and direction, although activity can be recorded from corticospinal neurons during uncomplicated walking (Armstrong and Drew 1985; Bretzner and Drew 2005; Drew 1993). Likewise, noninvasive human experiments using transcranial magnetic stimulation (TMS) have provided evidence that the excitability of corticospinal pathways to leg and arm muscles is modulated during the gait cycle and that corticospinal drive contributes directly to the activation of the muscles during uncomplicated treadmill walking (Barthélemy and Nielsen 2010; Capaday et al. 1999; Petersen et al. 1998b, 2001; Schubert et al. 1997). Lesion of the corticospinal tract has been shown to be associated with inadequate paw lift during gait in the cat (Jiang and Drew 1996) and monkey (Courtine et al. 2005) and it is generally believed that foot drop following stroke and spinal cord injury (SCI) in human subjects is also explained by impaired corticospinal transmission or lesion to the corticospinal tract (Calancie et al. 1999; McKay et al. 2005; Nathan 1994; Thomas and Gorassini 2005). However, since stroke and SCI generally involve many other pathways in addition to the corticospinal pathways there is only circumstantial evidence to support this. In the present study we therefore addressed whether foot drop in patients with SCI is correlated to measures of corticospinal transmission such as the latency and amplitude of motor-evoked potentials (MEPs) and intramuscular coherence analysis.

Cross-correlation and coherence analysis of paired electromyographic (EMG) recordings from the tibialis anterior (TA) muscle have been shown to provide evidence of modulation of the common synaptic drive to populations of TA motoneurons during walking (Halliday et al. 2003). Coupling of motor unit activities in the $10-$ to $20-\mathrm{Hz}$ frequency band is strongly reduced in persons with SCI, suggesting that motor unit synchrony during walking depends on an intact supraspinal drive to the spinal cord (Hansen et al. 2005). With similar findings of reduced $10-$ to $20-\mathrm{Hz}$ coherence in the TA of stroke patients, the evidence supports the view that the loss of coherence during walking is associated with a deficit in motor drive from 
the corticospinal tract (Nielsen et al. 2008). Hansen et al. (2005) found no relation between reduced TA motor unit coherence and the speed of gait in SCI persons. One explanation is that corticospinal deficits may be better correlated to specific deficits in the walking pattern rather than in the control of the step period.

Thus we show herein that a relationship can be established between parameters that may reflect corticospinal function (coherence, motor unit synchronization, and MEPs elicited by TMS) and the physical foot drop deficit that is observed following SCI. Each of these parameters is accessible through simple electrophysiological testing and, if proven reliable, could be adopted within a clinical setting for patient monitoring and follow-up. Part of this study was previously presented in abstract form (Barthélemy et al. 2008).

\section{METHODS}

The experimental protocol for SCI and control participants was approved by the local ethics committee and was in accordance with the Declaration of Helsinki. Participants received oral and written information and then gave their written consent to the study.

\section{Participants}

SPINAL CORD INJURED PARTICIPANTS. In all, 24 participants with a chronic SCI (22 men and 2 women; average age: $43 \pm 14 \mathrm{yr}$; range: 20-66 yr) were recruited from the Clinic for Spinal Cord Injuries, Rigshospitalet in Denmark. Inclusion criteria were that the injury (traumatic or nontraumatic) must have been stable for $\geq 1 \mathrm{yr}$ prior to the experiment and the participants must not have any known peripheral neuropathies or injuries. The participants had locomotor abilities (ASIA [American Spinal Injury Association] Impairment Scale [AIS] D) and were able to walk a distance of $10 \mathrm{~m}$ with or without assistance. One participant was categorized as AIS A, due to lack of sacral sparing (Marino et al. 2003), although he could still walk and was otherwise equivalent in motor function to an AIS D participant. $\mathrm{He}$ was therefore included in this study. The average number of years since the SCI participants had their injury was $12 \mathrm{yr}$ ( $\pm 11 \mathrm{yr}$ ) prior to the experiment. Nineteen of 24 SCI participants had a cervical lesion, 4 had a thoracic lesion, and one had a lesion at the first lumbar level. Ten SCI participants used a cane or crutch on short to medium distances and 14 walked unaided on all distances. Eleven SCI participants took daily medication: 4 took medication against pain, 2 took medication for bladder control, and 5 were taking antispastic medication on a regular basis.

CONTROL PARTICIPANTS. Fifteen age- and gender-matched neurologically healthy participants (13 men and 2 women; average age: $42 \pm 16 \mathrm{yr}$ ) took part in this study. None of the control (CTRL) participants had any history of neurological disease.

\section{Experimental protocol}

CLINICAL ASSESSMENT. A clinical assessment of the SCI participants was performed at the Clinic for Spinal Cord Injuries, Rigshospitalet in Hornbaek, Denmark. A trained physical therapist assessed the 10-m walk test following guidelines of Spinal Cord Injury Rehabilitation Evidence (SCIRE; www.icord.org/scire/home.php). Overground speed was determined based on that test: the distance $(10 \mathrm{~m})$ was divided by the time it took (in s) for the participant to walk that distance at their preferred speed.

A physiatrist performed the Standard Neurological Classification of Spinal Injury assessment including the AIS grade and, from these assessments, determined the spinal level of the lesion (Marino et al. 2003).

ELECTROPHYSIOLOGICAL ASSESSMENT. EMG activity was recorded from the TA and soleus muscles of both legs. On the most impaired leg, two pairs of TA EMG electrodes were placed over the muscle, with a minimum of $10 \mathrm{~cm}$ separating each pair (Halliday et al. 2003; Hansen et al. 2001). The signals were amplified ( $\times 1,000-5,000)$, band-pass filtered (5-25 to $1,000 \mathrm{~Hz}$ ), then digitized and sampled (2 $\mathrm{kHz}$ ) to a computer using a Micro1401 interface (Spike and Signal software; Cambridge Electronic Design, Cambridge, UK).

MEASUREMENT OF MAXIMAL M WAVE AND MAXIMAL VOLUNTARY CONTRACTION OF TIBIALIS ANTERIOR. To compare MEP and EMG amplitudes in the TA of all participants, we expressed them as a percentage of the maximal compound muscle action potential (Mmax) elicited by a supramaximal electrical stimulation.

Mmax was evoked in TA by stimulating the common peroneal nerve $(\mathrm{CPN})$ at the level of the head of the fibula. The participants were seated with a hip angle of $90-100^{\circ}$ and knee and ankle angles of $90^{\circ}$. CPN stimulation consisted of a 1-ms rectangular pulse delivered by a constant-current stimulator (1-1,000 mA, DS7A; Digitimer, Welwyn Garden City, Hertfordshire, UK). The strength of the stimulation was increased until no further changes in the amplitude of the M-wave were observed. The final value was noted as Mmax.

The maximal isometric voluntary contraction (MVC) of TA was recorded in the same position. The foot was firmly stabilized on a custom-made foot rest, which ensured that all contractions were isometric when dorsiflexion was performed. Instantaneous feedback was provided to the participant by displaying on an oscilloscope placed in front of the participant the amplitude of the rectified, integrated (time constant: $200 \mathrm{~ms}$ ), EMG activity produced by TA. Three trials were used for the MVC. The trial that produced the highest value was noted and later used to determine the level of contraction the participant had to produce during subsequent measurements (see the following text). Thus in this study the participants were asked to hold a constant level of background EMG contraction during the TMS stimulation. However, Davey et al. (1999) reported that the pattern of force contraction and MEP response in the SCI participants are very different from those of control participants and thus the force of contraction does affect the response of the TMS stimulation. Similar phenomena could be inherent with the technique used in this project and have been addressed, by constant visual feedback given to the participants, and rest between trials were taken regularly to minimize fatigue.

CORTICOSPINAL STIMULATION. To assess transmission in the corticospinal tract (CST), we used TMS (Magstim Rapid Rate stimulator; Magstim, Dyfed, UK). The stimuli were applied over the leg area of the motor cortex contralateral to the leg tested by using an $80-\mathrm{mm}$ double-cone coil (\#9902-00). The optimal position for evoking MEPs in TA was determined and the coil was subsequently held over this site by an experimenter, with the handle directed toward the back, such that the induced current flowed in a posterior to anterior direction in the brain. Overall, this location was slightly lateral to the vertex (contralateral to the side under investigation). The motor threshold (MT) of the MEP was determined as the stimulus intensity at which three of five stimuli evoked a recognizable MEP, i.e., a response $>50$ $\mu \mathrm{V}$ at rest and 100-200 $\mu \mathrm{V}$ during contraction (Rothwell et al. 1999).

TMS was applied with the participants seated as described previously during ankle dorsiflexion equivalent to $10 \% \mathrm{MVC}$ and at rest. Both legs were tested, starting with the most impaired side. Stimulation was applied 5 to 10 times at $1.2 \times$ MT.

GAIT ABILITY. Gait ability was assessed in all participants while they were walking on a treadmill (TecnoGym with minimum speed $0.2 \mathrm{~m} / \mathrm{s}$ ). SCI participants did not wear any orthoses during the assessments. A security harness was used to mitigate against the risk of falls in all SCI participants who were unable to walk without a 
walking aid. The harness was secured by a Biodex support system, but did not provide any active body weight support. SCI participants could also hold on to handrails if they found it was necessary.

The kinematics of locomotor movements was analyzed using a six-camera Pro-reflex Qualisys system (Qualisys, Gothenburg, Sweden). Passive markers were placed on both legs and were positioned on the anterior superior iliac spine, greater trochanter, external joint line of the knee, external malleolus, and the lateral side of the 5th metatarsophalangeal joint (MTP). Markers were also placed on the 12 th thoracic spinal process, the 7 th cervical spinal process, and one on each acromion.

All participants wore a pressure-sensitive resistance sensor under the heel of the most impaired leg to monitor the time of heel contact during each step cycle. The heel contact times were then used as triggers during data analysis (see following text).

A target of $5 \mathrm{~min}$ of treadmill walking was set for each participant. SCI participants walked at the maximal speed at which they were comfortable without holding the bars if possible. CTRL participants were asked to walk with different speeds (their own favorite speed and speeds comparable to those of SCI participants). Kinematics and EMG data collection were synchronized and during the 5-min walking trial at least two sets of 1-min data were recorded.
Locomotion of the participants was also recorded with a video camera (model HDRSR12; Sony).

\section{Data analysis}

KINEMATICS AND EMG RECORDINGS. The kinematic recordings from the infrared cameras were preanalyzed in Qualisys software, where the different markers were identified. Periods of 30-60 s of kinematic measurements were then exported from Qualisys to custom-made software (Matlab and Excel-based), which calculated average swing length and durations of swing, of stance, and of the gait cycle. Figure 1, illustrates examples of these analysis for one CTRL (Fig. 1, $A-D$ ) and two SCI participants (Fig. 1, $E-H$ and $I-L$ ). The stick diagram of leg movement was reconstructed from marker positions for each participant (an example of this is shown in Fig. 1, A, E, and $I$ ). The amplitude of toe elevation at the onset of swing (small vertical line) and just prior to heel strike (first and second toe-lifts, respectively; Fig. $1, B, F$, and $J$ ) as well as angle excursion of knee and ankle (Fig. 1, $C, G$, and $K$ ) were calculated. Corresponding EMG recordings from TA and soleus are shown in Fig. 1, $D, H$, and $L$.
A
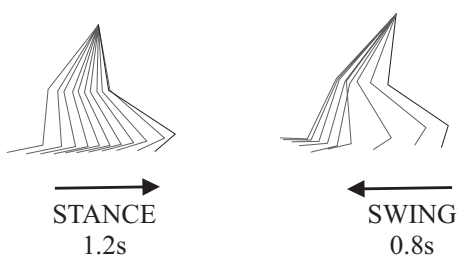

B

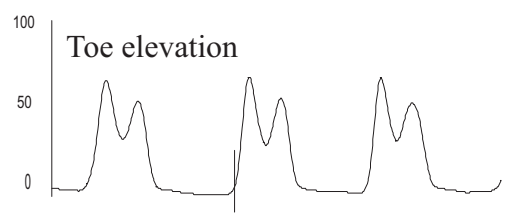

\section{C}

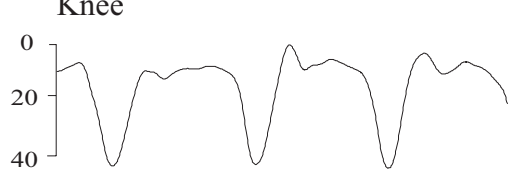

Ankle

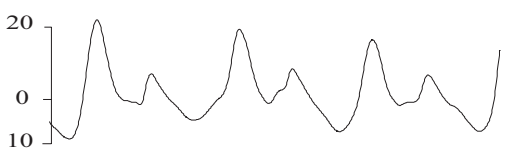

TA

D
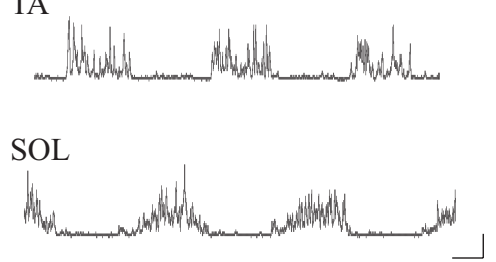

E

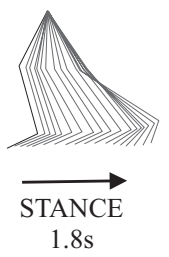

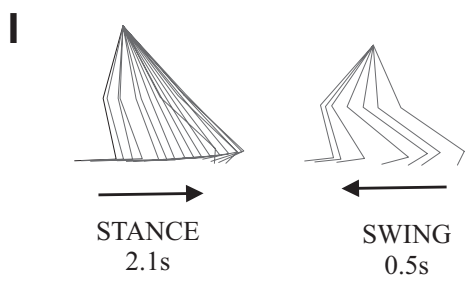
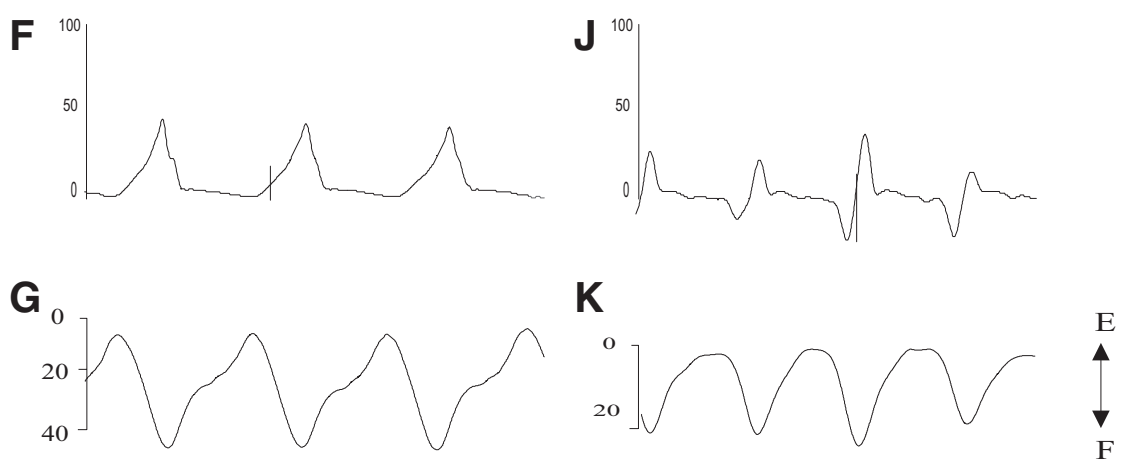

F
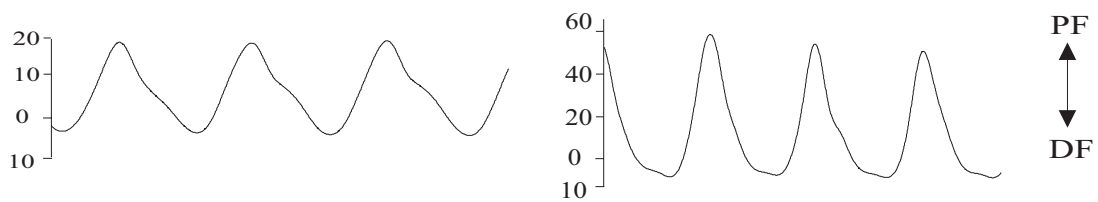

H
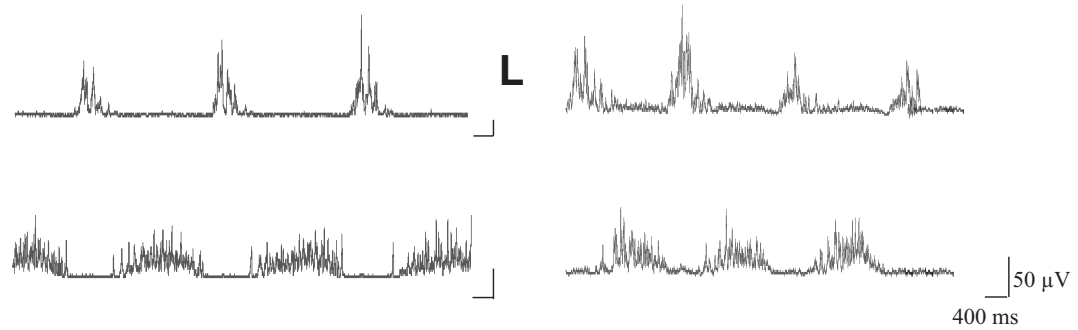

FIG. 1. Walking pattern. Kinematic analysis of the gait pattern of a control participant $(A$ to $D)$ and 2 spinal cord injured (SCI) participants $(E$ to $H$ and $I$ to $L$ ). Stick figures show the leg at different time points during stance and swing phase $(A, E$, and $I)$. Excursion of the toe marker, knee, and ankle angle pattern and electromyographic (EMG) activity of tibialis anterior (TA) and soleus (SOL) muscles are displayed for all 3 participants. The vertical line in the toe marker plots points to the onset of the elevation of the toe. 
FOOT DROP MEASURES. Toe elevation. The elevation of the toe above ground level was taken as a measure of foot drop. However, trying to determine objective measures for foot drop was complicated by the compensations that are taking place at other articulations and that are different from one participant to the other. Trying to calculate each of those compensations and subtracting them systematically in every participant was not conclusive. Therefore the amplitude of toe elevation was calculated from the distance between the marker placed on the MTP and the ground. The largest distance between these two points was determined and will be referred to as the highest toe elevation. In healthy participants plotting the elevation against time reveals the presence of two separate peaks of toe elevation during swing. The presence or absence of the second toe elevation prior to heel strike was also determined by subtracting the excursion of the malleolus marker (which can be caused by flexion of the knee, hip, or other compensation strategies used by the SCI participants) from the elevation of the toe. The remaining movement would then mainly occur at the ankle at a time corresponding to the second toe elevation.

Angular difference. Simply measuring the total angle excursion would have been misleading since some SCI participants restricted their movement at the ankle, whereas others dragged their foot, which increased the total excursion. Furthermore, it would not have been specific to the part of the cycle where foot drop is the most evident: the onset of swing. Therefore to address the decreased toe elevation of the first part of the swing, the ankle excursion between toe-off and midswing was calculated. Indeed, the angle of the ankle was measured at toe-off, just prior to the foot being lifted from the ground. At that time, the ankle is at its most extended and, to keep the foot from touching the ground at the onset of the forward movement, there is dorsiflexion that raises the toe until midswing. This constitutes the first part of the swing and corresponds to the first toe elevation. For each participant, the ankle angle at midswing was calculated and then subtracted from the angle measured at toe-off. For CTRL participants (walking at speeds comparable to those of SCI participants), this angle excursion had a mean of $16 \pm 4^{\circ}$. For SCI participants, this angle excursion was quite varied and could be smaller or larger from this mean depending on the foot drag, irrespective of whether they could correct for it or whether they prevented their foot to drag by lifting the foot prematurely at the end of stance. The difference between the values of the angle excursion at onset of swing for each subject was subtracted from the mean value of the CTRL participants. The absolute value of this measure will be referred to as angular difference and, in that way, subjects with low angular difference are closer to the mean value observed in control.

MEPS. The MEP amplitude was measured at rest by taking the peak-to-peak value of each MEP, averaging those values, and expressing them as percentage of Mmax. Although in iSCI participants MEP could lack a clear biphasic shape due to the late components of the corticospinal inputs, we concentrated on the amplitude of the highest components of the MEP. The latency was measured during contraction equivalent to $10 \% \mathrm{MVC}$, using the rectified and averaged MEP. The latency corresponded to the onset of the first significant deviation from the background EMG.

It would have been optimal to subtract an estimate of peripheral conduction time in each of the subjects to obtain a more precise estimate of central conduction time. However, most of the subjects did not tolerate magnetic stimulation applied over the lumbar spinal cord and this procedure therefore had to be abandoned. We did make sure to exclude subjects with known peripheral neuropathy and/or diabetes and differences in peripheral conduction time therefore likely had only little influence on differences in MEP latency. Normalization of the MEP latency to the height of the subjects also made no difference for the comparison of CTRL and SCI subjects or for the correlation of MEP latency in different subjects to other parameters, suggesting that differences in height between subjects was too little (1.64 to $1.84 \mathrm{~m}$ ) to significantly influence the distribution of MEP latencies.
COHERENCE AND CROSS-CORRELATION. Analysis of the inputs (e.g., CST) responsible for driving spinal motoneurons during movement can be obtained from coherence and cross-correlation analysis of EMG activity (Halliday et al. 1995; Hansen et al. 2001, 2005). The presynaptic inputs that synchronize the populations of motor units can be estimated by doing a statistical analysis and by characterizing their frequency content and time course.

The method uses the Neurospec scripts available online (www. neurospec.org) and has been described elsewhere (Hansen et al. 2005; Nielsen et al. 2008). Briefly, a Fourier transform-based framework is used for the analysis. A correlation structure between the paired EMG signals can then be characterized as a function of time and frequency (Halliday et al. 1995). Standard practice and the theoretical work of Myers et al. (2003) were followed. Surface EMG was preprocessed using a full-wave rectification. The rectified signals were then assumed to be realizations of stationary zero mean time series, which were denoted by $x$ and $y$. A periodogram was applied to estimate the power spectra. From these spectra a discrete Fourier transform was constructed from short sections of the data taken at a fixed offset time with respect to a trigger point (in this case the heel strike) in each step cycle. By an average of the periodograms across all step cycles estimates of the spectra were constructed. For the data presented, a segment length corresponding to the period of TA EMG activity during the swing phase was used. $f_{x x}(\lambda)$ and $f_{y y}(\lambda)$ were used to represent the power spectra of processes $x$ and $y$, respectively. The cross-spectrum between $x$ and $y$ was denoted by $f_{x y}(\lambda)$ and was estimated in a manner similar to that of the autospectra. The correlation between the EMG signals was assessed through coherence functions in the frequency domain (Brillinger 1981; Halliday et al. 1995). The coherence function between the two rectified EMG signals was defined at frequency $\lambda$ as

$$
\left|R_{x y}(\lambda)\right|^{2}=\frac{\left|f_{x y}(\lambda)\right|^{2}}{f_{x x}(\lambda) f_{y y}(\lambda)}
$$

On a scale from 0 to 1 , coherence functions provide normative measures of linear association. For the present data, the coherence provides a measure, at each Fourier frequency $\lambda$, of the fraction of the activity in one surface EMG signal that could be predicted by the activity in the second surface EMG signal. In this way, the coherence was used to quantify the strength and frequency of common rhythmic synaptic inputs, which were distributed across the motoneuron pool (Farmer et al. 1993). To correlate coherence measures to other measurements in this study, the area under the curve was calculated in frequency windows determined to be different between CTRL and SCI participants $(10-20$ and $20-50 \mathrm{~Hz})$.

In the time domain, estimates of the cumulant density function were used to characterize the correlation between the two rectified surface EMG signals. The cumulant density function, denoted by $q_{x y}(u)$, was defined as the inverse Fourier transform of the cross spectrum

$$
q_{x y}(u)=\int_{-\pi}^{\pi} f_{x y}(\lambda) e^{i \lambda u} \mathrm{~d} \lambda
$$

For two uncorrelated signals, the cumulant has an expected value of zero; deviations from this indicate a correlation between the two EMG signals at a particular time lag, $u$. In the present experiments, which are exploring aspects of the common drive to TA motoneurons during locomotion, the dominant feature in cumulant density estimates is the central peak around zero, reflecting the presence of common synaptic input. Rhythmic inputs will induce symmetrical oscillatory components in the cumulant (Perkel et al. 1967a,b); the frequency and strength of these components can be quantified from the corresponding coherence estimates. Cumulant density functions are analogous to cross-correlation functions often used to quantify spike train data and have a similar interpretation (Halliday et al. 1995). 


\section{Statistics}

The two groups in this study (SCI vs. CTRL) were compared for TMS responses, coherence, kinematic parameters, and gait velocity. Student's $t$-test was applied to determine significance of the comparison, but in cases where distribution of data was not normal or not of equal variance, the nonparametric Mann-Whitney rank-sum test was applied. To further the analysis, correlations were made between electrophysiological, clinical, and biomechanical tests. The Pearson product moment method was used to measure the correlation for all parameters except those that did not follow a normal distribution, which were assessed with the Spearman rank-order test. In the graphs, a regression line was also applied for visualization purposes. Statistical analysis was performed using SigmaPlot 11.0 and, for all tests, a difference was considered significant if $P<0.05$. A Bonferroni correction was applied when numerous correlations were made between coherence, kinematics, MEP measures, and gait speed. Averages are presented as mean $\pm \mathrm{SD}$.

\section{R E S U L T S}

\section{Comparison of gait characteristics related to foot drop in CTRL and SCI participants}

The kinematics and EMG activity shown in Fig. 1 illustrate the differences in gait ability in one CTRL (Fig. 1, $A-D$ ) and two SCI participants walking on a treadmill (Fig. 1, E-L). CTRL participants walking at speeds comparable to those of SCI participants (from 0.2 to $0.8 \mathrm{~m} / \mathrm{s}$ ) and at their own comfortable pace (1-1.1 $\mathrm{m} / \mathrm{s}$ ) demonstrated two peaks of toe elevation during the swing phase. Toe elevation is shown as an upward deflection in the graphs in Fig. $1, B, F$, and $J$. The first elevation starts at the beginning of swing (see the lift in the rightmost part of stick figure in Fig. 1A) and the second elevation occurs at the end of the swing, prior to the heel strike (leftmost part). The average amplitude of the toe elevation for the CTRL participants was $59 \pm 15 \mathrm{~mm}$ for the first and $67 \pm 20 \mathrm{~mm}$ for the second toe elevation when walking at an average speed of $0.6 \mathrm{~m} / \mathrm{s}(0.2-0.8 \mathrm{~m} / \mathrm{s})$. No significant difference in the toe elevation was found when CTRL participants walked at their own comfortable speed.
Thirteen SCI individuals also showed two toe elevations in the swing phase during gait (average highest peak of $74 \pm 17$ $\mathrm{mm}$ ), whereas 11 individuals showed only a single elevation (similar to the participant in Fig. 2, $E-H$; average height $31 \pm$ $15 \mathrm{~mm}$ ). Seven of these dragged their foot at the onset of swing (exemplified by the participant in Fig. $1, I-L$ ). The movement at the ankle joint also shows two dorsiflexions during the cycle for CTRL, but not for SCI corresponding to the absence of the second toe elevation during treadmill walking (Fig. 1, C, G, and $K)$. Total range of ankle movement was not significantly different between the CTRL and SCI groups $(25 \pm 5$ and $25 \pm$ $13^{\circ}$, respectively; rank-sum test, $\left.P=0.07\right)$. Furthermore, there was no statistical difference when CTRL participants were compared with SCI subjects who exhibited foot drag (30 \pm $23^{\circ}$; rank-sum test, $P=0.3$ ), but the variability was greater.

\section{Modulation of common motoneuronal drive during gait in CTRL and SCI participants}

Figure 2 shows coherence and cumulant analysis data from CTRL (Fig. 2, $A-C$ ) and SCI participants (Fig. 2, $D-F$ ) walking at $0.4 \mathrm{~m} / \mathrm{s}$, respectively. Two clear bursts of EMG activity were observed in each TA recording for the CTRL participant, corresponding to the first and second toe elevations, but only one burst was observed in the EMG recording of the SCI participant. In all participants, a very high coherence between the two TA EMG recordings was observed at frequencies $<10 \mathrm{~Hz}$. This is assumed to be produced, in part, by the common envelope of EMG activity during swing (Halliday et al. 2003). In agreement with what has been reported previously (Halliday et al. 2003; Hansen et al. 2005), an additional peak of coherence was observed in the CTRL participants at frequencies around $10-20 \mathrm{~Hz}$ and extending out to $50 \mathrm{~Hz}$ (Fig. 2B), but not in the SCI participant (Fig. 2E). In Fig. 2, $C$ and $F$, the corresponding cumulant density provides a measure of the temporal structure of the motor unit synchronization existing between paired EMG recordings for the duration of the swing phase. A narrow central peak of synchronization (last-

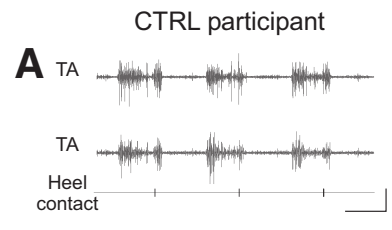

B
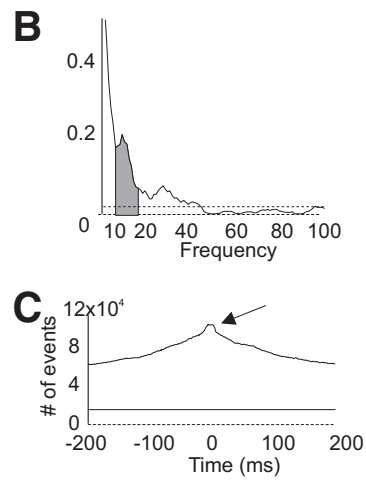

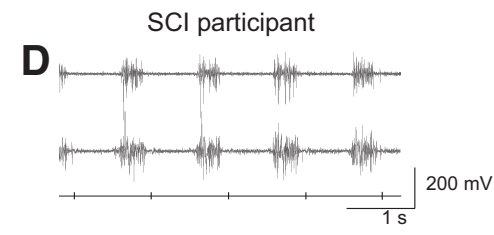

E

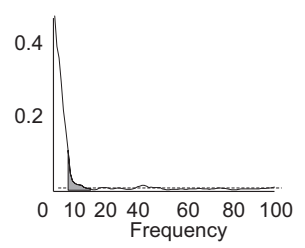

$\mathbf{F}$

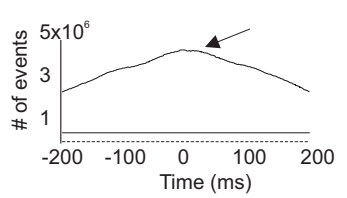

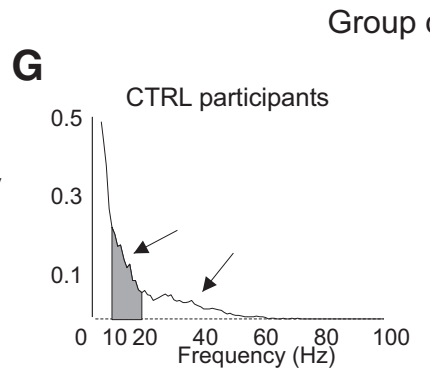

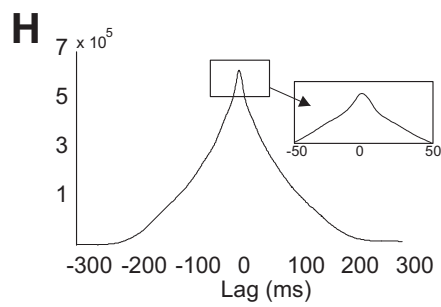

FIG. 2. Cross-correlation and coherence analysis. A: EMG pattern of both recordings along the TA muscle are displayed for a control (CTRL) participant

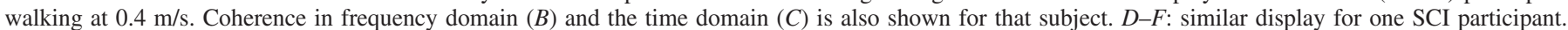

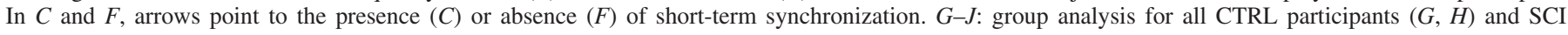
participants $(I, J)$. In $G$, arrows point to the presence of coherence in the 10 - to 20 - and 20 - to $50-\mathrm{Hz}$ frequency windows. 

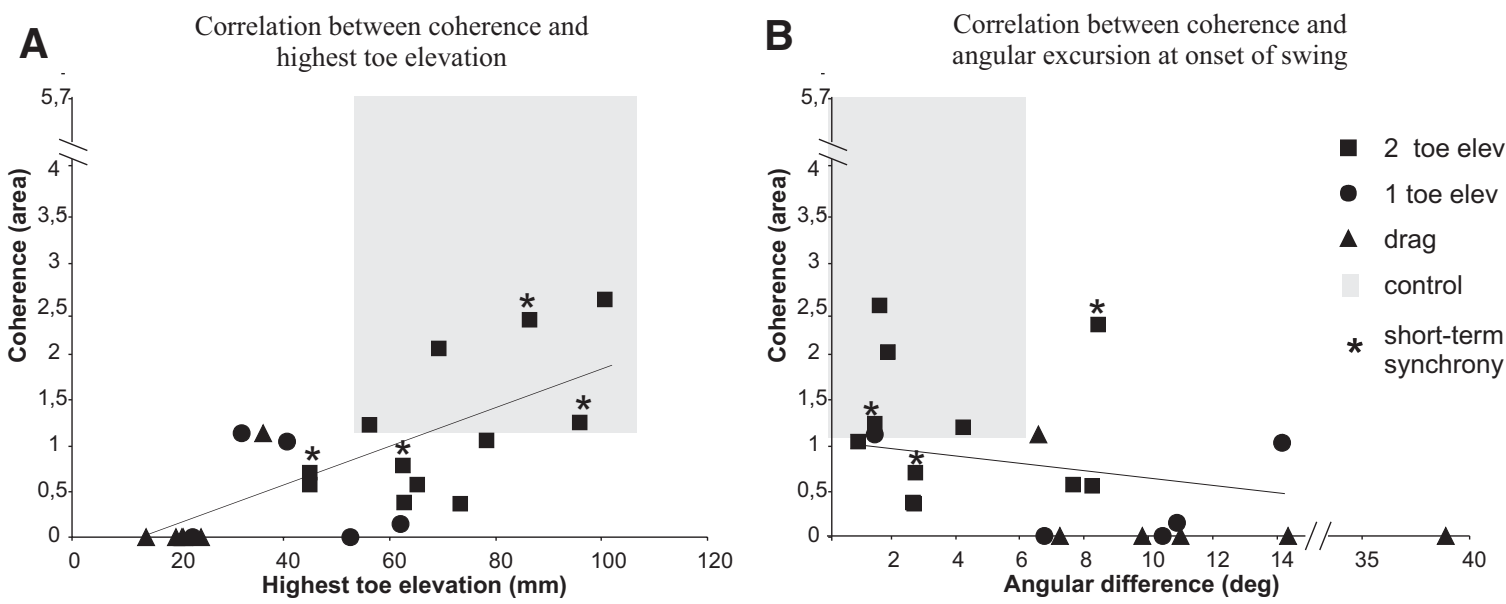

FIG. 3. Correlation between coherence and foot drop measures. Correlation between the highest toe elevation and coherence during walking sequence on a treadmill. The squares represent SCI participants displaying 2 toe elevations during swing. The circles represent participants with one toe elevation and the triangles represent the participants with only one toe elevation and who are dragging their foot on the floor at the onset of swing. The shaded area represents the values for control participants. An asterisk is annexed to the SCI participants where short-term synchrony in the time domain was observed.

ing about $23 \mathrm{~ms}$ ) was observed in the cumulant density estimate of the CTRL participant. This sharp central peak is thought to result from the common central drive to the motoneurons. In the SCI participant, the central peak was absent and a broad low-amplitude peak at a lag of $0 \mathrm{~ms}$ and width of almost $90 \mathrm{~ms}$ was observed.

Figure 2, $G-J$ shows pooled data analyzed from 15 CTRL and 23 SCI participants. The EMG data pooling relates to the TA activity sampled prior to heel strike and for both SCI and CTRL participants has a duration of $600 \mathrm{~ms}$. Pooled coherence for CTRL participants contains two zones of significant coherence $>10 \mathrm{~Hz}$ and occurring within the 10 - to 20 - and 20- to $50-\mathrm{Hz}$ frequency bands. Both of these zones of coherence are lacking from the pooled coherence of SCI participants. The pooled cumulant density is shown over two lag ranges of \pm 50 and $\pm 300 \mathrm{~ms}$. The narrow central peak synchronization was evident for CTRL participants, but only a larger, broader peak of synchronization was present for the SCI participants.

\section{Correlation of strength of common synaptic drive and foot drop in SCI individuals}

Figure $3 A$ shows that the amount of coherence in the 10 - to $20-\mathrm{Hz}$ frequency band was positively correlated (Spearman $r=$
$0.7 ; P=0.0004)$, with the highest toe elevation in individual SCI participants. It is also seen that participants displaying two toe elevations (squares) had higher toe elevation than that of participants who displayed a single toe elevation (circles) and who dragged their foot at the onset of swing (triangles). For comparison the range of highest toe elevation in CTRL participants was 52-109 $\mathrm{mm}$ and area of coherence went from 1.2 to 5.7. The data from the CTRL participants are shown as the shaded gray area. There was a significant negative correlation between the angular difference at the onset of swing (range in CTRL: $0-6^{\circ}$ ) and the amount of coherence in the $10-$ to $20-\mathrm{Hz}$ frequency range (Fig. $3 B$; Spearman $r=-0.62 ; P=0.008)$. There was also a significant correlation between coherence in the 20- to $50-\mathrm{Hz}$ frequency band and the highest toe elevation (Spearman $r=0.5 ; P=0.04$ ), but not with the angular difference (Spearman $r=-0.5 ; P=$ 0.08). Only four SCI participants showed short-term synchrony in the cumulant density function (marked with asterisks).

\section{Transmission in CST evaluated by TMS}

Figure 4 shows sample averaged TA MEPs at rest in a representative CTRL (Fig. $4 A$ ) and the most affected leg of an SCI individual (Fig. 4B). MEPs could be evoked at rest in all
A $\quad$ CTRL

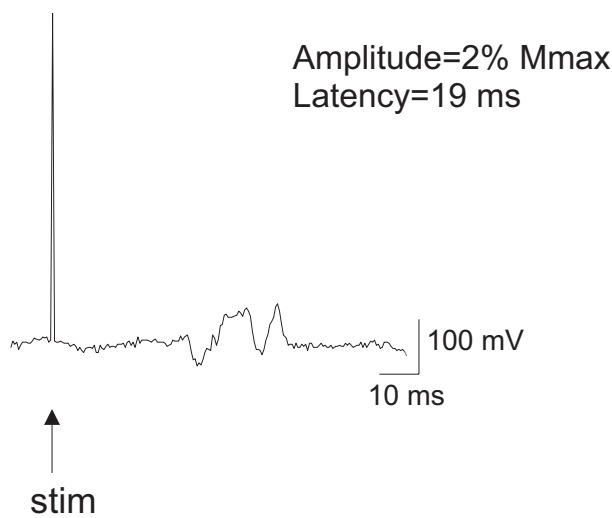

B $\quad \mathrm{SCl}$

No MEP at rest

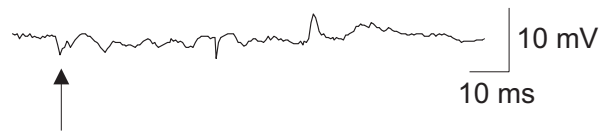

FIG. 4. Motor-evoked potentials (MEPs) in a CTRL and SCI participant. MEP at rest of TA muscle in CTRL $(A)$ and SCI participants $(B)$. Arrows point to the time of stimulation. 
TABLE 1. Correlations between coherence, motor-evoked potential (MEP), and kinematics in the most impaired leg of spinal cord injury (SCI) participants

\begin{tabular}{|c|c|c|c|c|}
\hline $\mathrm{r} / \mathrm{P}$ & Highest Toe Elevation & Angular Difference & \multicolumn{2}{|c|}{ MEP } \\
\hline $20-50 \mathrm{~Hz}$ & $0.5 / 0.04 *$ & $-0.5 / 0.08$ & $0.38 / 0.96$ & $-0.3 / 1$ \\
\hline
\end{tabular}

$r$, correlation factor; $P, P$ value. $* P<0.05 ; * * P<0.01 ; * * * P<0.001$

13 CTRL individuals tested with TMS, whereas it was not the case for 6 SCI participants. The threshold of the MEP was significantly lower in the CTRL than that in the most affected leg of the SCI individuals $(64 \pm 12 \%$ MSO [maximal stimulator output] for CTRL and $81 \pm 19 \%$ MSO for SCI; Student's $t$-test, $P=0.007)$ and the MEP amplitudes were greater in CTRL than those in SCI participants $(7 \pm 5 \% \mathrm{Mmax}$ for CTRL vs. $4 \pm 6 \% \mathrm{Mmax}$ for SCI; rank-sum test, $P=0.04)$. During dorsiflexion at $10 \% \mathrm{MVC}$, MEPs were evoked in all CTRL and SCI individuals. The threshold was $55 \pm 11 \% \mathrm{MSO}$ for CTRL and $67 \pm 15 \%$ MSO for SCI (Student's $t$-test, $P=0.02$ ). The MEPs in SCI participants had a significantly longer latency $(40 \pm 6 \mathrm{~ms})$ than that in CTRL participants (latency: $32 \pm$ $2 \mathrm{~ms}$; rank-sum test, $P<0.001)$.

Importantly, the SCI group was heterogeneous with 5 SCI participants displaying MEPs during contraction and at rest of similar latency and amplitude to CTRL participants, 13 having significantly longer latency and/or smaller amplitude both at rest and during contraction and 6 having significantly reduced and delayed MEPs during contraction but no MEPs at rest.

\section{Correlation of strength of common motoneuronal drive,} corticospinal transmission, and foot drop

MEP measurements during rest and contraction were not significantly correlated to the amount of coherence in either the 10- to 20 - or the $20-$ to $50-\mathrm{Hz}$ range (see Table 1 ).

Correlations between kinematics and MEP measures were assessed using data from both legs (Table 2). The highest toe elevation was significantly correlated to the MEP latency and the MEP amplitude at rest (Fig. 5, $A$ and $B$; Spearman $r=$ $-0.5, P=0.04$ and $r=0.6, P=0.01$, respectively).

\section{Correlation with overground gait speed}

Highest toe elevation and angular difference in early swing measured in the most impaired leg were directly correlated to overground gait speed, based on the 10-m test (Table 3; Spearman $r=0.6, P=0.006$ and $r=-0.5, P=0.02$, respectively). MEP amplitude at rest was significantly correlated to gait speed (Spearman $r=0.7 ; P=0.009$ ), so that SCI

TABLE 2. Correlations between kinematics and MEPs in SCI participants

\begin{tabular}{lcc}
\hline \hline \multirow{2}{*}{$\mathrm{r} / \mathrm{P}$} & \multicolumn{2}{c}{ MEP } \\
\cline { 2 - 3 } & Latency Contraction & Amplitude at Rest \\
\hline Highest toe elevation & $-0.5 / 0.04^{*}$ & $0.6 / 0.01^{* *}$ \\
Angular difference & $0.2 / 1$ & $-0.3 / 0.6$ \\
\hline
\end{tabular}

$* P<0.05 ; * * P<0.01 ; * * * P<0.001$. participants who did not show any MEPs at rest, had the slowest gait speed overground. MEP latency and coherence were not significantly correlated to overground gait speed.

\section{I S C US S I O N}

Herein, we show that coherence between populations of TA motor units, MEP amplitude at rest, and MEP latency during contraction are correlated to the degree of foot drop in indi-
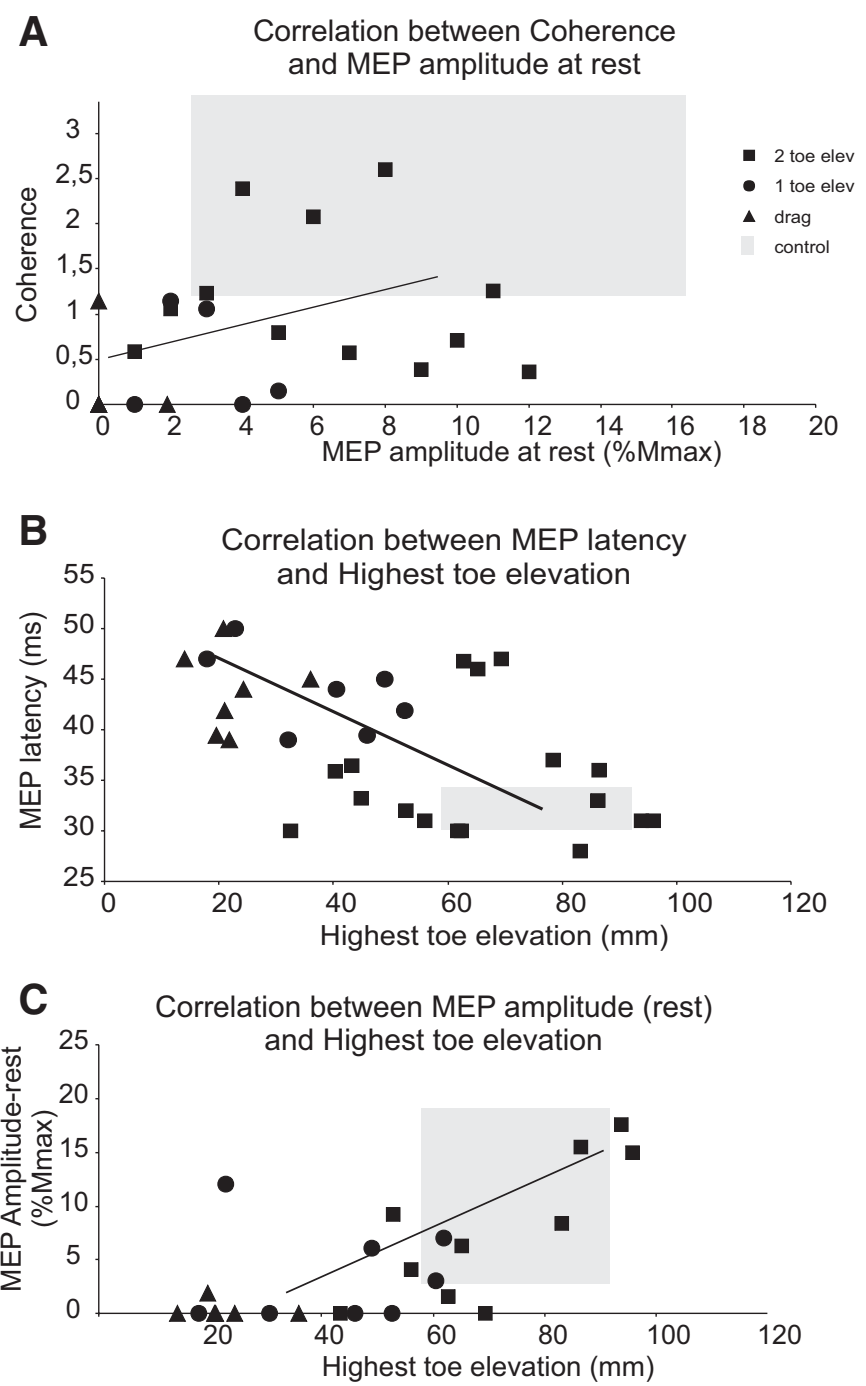

FIG. 5. Correlations between MEP, coherence and kinematic measures. In $A$, coherence is plotted against MEP amplitude assessed at rest. Each point represents a participant. In $B$, MEP latency is correlated to the highest toe elevation and in $C$, MEP amplitude at rest is correlated to highest toe elevation. For $B$ and $C$, both legs of the participants were analyzed. 
TABLE 3. Correlations between coherence, MEP measurements, kinematics, and gait speed in SCI participants

\begin{tabular}{|c|c|c|c|c|c|c|}
\hline $\mathrm{r} / \mathrm{P}$ & $10-20 \mathrm{~Hz}$ & $20-50 \mathrm{~Hz}$ & \multicolumn{2}{|c|}{ MEP } & Highest toe Elevation & Angular Difference \\
\hline
\end{tabular}

$* P<0.05 ; * * P<0.01 ; * * * P<0.001$.

viduals with SCI. This suggests that transmission in the corticospinal tract (CST) to TA motoneurons is of functional importance for lifting the foot during the swing phase of human gait. In addition, intramuscular coherence in TA may serve as a physiological marker of impaired corticospinal control of gait in SCI participants.

\section{Intramuscular coherence as a tool for assessment of CST impairment}

The two main characteristics of coupling between TA motor unit activity in the swing phase of healthy human walking i.e., a peak of coherence in the $10-$ to $20-\mathrm{Hz}$ frequency band and synchronization within a narrow time band $(\sim 20 \mathrm{~ms})$ were not seen in SCI participants, as previously reported by Hansen et al. (2005). However, they found no correlation between the motor unit coupling and the gait ability of the subjects (i.e., speed of walking). In the present study, we show that the coherence was well correlated with the degree of foot drop in the SCI participants and that foot drop in turn was well correlated to the speed of walking. Lack of correlation between gait speed and coherence can be explained by the fact that coherence in TA is assessed only during swing phase activity, whereas gait speed depends not only on swing phase activity but also on other variables, such as stance phase activity and transitions from swing to stance.

Cross talk between the two surface electrode recordings is unlikely to explain the data observed, as discussed in Hansen et al. (2001, 2005) and Halliday et al. (2003). The distance between the pairs of EMG electrodes exceeds that of the overlap between individual motor unit territories (Roy et al. 1995; Smits et al. 1994). Also, signs of cross talk within the cumulant (large, narrow central peak) or coherence (large broad band coupling at minimal phase lags) estimates were not detected (see Fig. 2).

It is generally accepted that common synaptic drive from collaterals of last-order neurons is an important, but probably not exclusive, contributor to short-term synchrony and coherence. Halliday et al. (2003) argued that coherence in the 10- to $20-\mathrm{Hz}$ frequency band during walking may reflect part of the discharge frequencies of the last-order neurons responsible for the common synaptic drive, just as was shown in the 15- to $35-\mathrm{Hz}$ frequency band during tonic voluntary contractions (Farmer et al. 1993,1997). Part of this common synaptic drive may be from corticospinal origin. Nielsen et al. (2008) showed a decrease in coherence between 10 and $20 \mathrm{~Hz}$ and an absence of a central sharp peak in the time domain in stroke patients. The reduction of the central synchronization peak and the 10to $20-\mathrm{Hz}$ coherence in the SCI participants included in the present study may similarly signify that CST activity is of importance for their occurrence. Indeed, the SCI participants in whom coherence and cumulant were diminished had a decreased elevation of the toe. Participants who demonstrated foot drop also had impaired corticospinal transmission, as evidenced by significantly increased latency during contraction and decreased amplitude of MEPs at rest. Coherence was also observed in 20 - to $50-\mathrm{Hz}$ bandwidth in healthy participants, but not in the most impaired SCI participants. This $20-$ to $50-\mathrm{Hz}$ coherence was significantly correlated to highest toe elevation measures and is thought to reflect the extent of corticospinal impairment after SCI. However, it could also be related to activity in the reticulospinal and/or propriospinal tracts, given that these pathways might contribute to recovery after spinal cord injury (Nishimura et al. 2009). Similarly, Norton and Gorassini (2006) showed an increase of coherence in a similar bandwidth $(24-40 \mathrm{~Hz})$ between muscles acting at the knee joint in SCI participants that improved their locomotor function following treadmill training.

Although both electrophysiological measures (TMS and coherence) were well correlated to foot drop, they were not significantly correlated to each other. TMS latency provides an estimate of impairment of the fastest conducting corticospinal neurons (Alexeeva et al. 1998; Brouwer et al. 1992; Calancie et al. 1999; Ravnborg 1996), whereas coherence in the 15- to $30-\mathrm{Hz}$ frequency band likely reflects common synaptic drive arising from a less well defined corticospinal activation (Datta et al. 1991; Farmer et al. 1993; Kilner et al. 1999; Nielsen et al. 2008). The diversity in the source of the signal might explain the lack of correlation between TMS and coherence and the two parameters are therefore unlikely to be equally influenced by a given spinal cord injury. It should also be pointed out that the two parameters were obtained in different tasks and that they likely reflect different aspects of corticospinal function.

\section{Involvement of the CST in foot drop}

Foot drop has long been associated with CST lesion. In the cat, a complete lesion of the CST induces dragging of the paw at the onset of swing (Drew et al. 2002; Jiang and Drew 1996). After spinal cord lesion, foot drop is also a permanent deficit in the monkey (Lawrence and Kuypers 1968). Human studies have also demonstrated that the CST contributes significantly to human walking (Capaday et al. 1999; Petersen et al. 1998a, 2001; Schubert et al. 1997) and TA appears to receive a very significant monosynaptic corticospinal drive (Petersen et al. 1998a,b, 2003). Clinically, foot drop is a frequent permanent functional deficit among individuals with SCI and stroke, which is consistent with a lesion to the corticospinal fibers along the path from the cortex, the cerebral capsule, the cerebral peduncles, and the spinal cord (Westhout et al. 2007). The present study strengthens this by showing that in SCI patients, a lesion disrupting the CST will induce foot drop and that the degree of the deficit is directly correlated to the extent of the CST impairment. 


\section{Measurement of foot drop}

It was not straightforward to obtain a valid comparable measure to estimate the extent of foot drop in the SCI participants from the kinematic data, since the subjects used different strategies to compensate for the foot drop. Previous studies have used a number of different measures to solve this problem, such as measuring total ankle motion range (Bensoussan et al. 2006), maximal dorsiflexion or plantarflexion (Novak et al. 2009), and ankle dorsiflexion at heel contact (Paoloni et al. 2009). We attempted using these measures but found them difficult to apply in relation to the gait characteristics observed in the subjects in the present study. We consequently used the following two parameters: 1 ) the highest elevation of the foot was measured as the total vertical excursion of the MTP marker. A similar measure has been used in several previous studies (toe clearance; Bensoussan et al. 2006; Chin et al. 2009; Weber et al. 2004) and was found to be simple to apply and to reflect well the degree of foot drop of the subject. The findings in the present study confirm this. 2) The angle excursion between toe-off and midswing was calculated to address the foot drop at the onset of swing. This value is relatively stable in CTRL participants, but analysis of SCI participant patterns revealed that they either decrease that amplitude (e.g., by lifting the foot early at the end of stance) or increase the amplitude (by dragging the foot on the ground). To circumvent this, the absolute difference between the mean angle excursion obtained with the CTRL and that of the different SCI participants was used. Both these parameters were correlated with clinical foot drop, with walking speed, and with each other.

\section{Clinical significance}

These results imply that reduction of coherence and shortterm synchrony may serve as physiological markers of impaired corticospinal control of gait in SCI subjects. Our results for the first time demonstrate a clear linkage between the behavioral deficits seen in ambulatory SCI persons and electrophysiological measures of corticospinal function. Importantly, each of these measures could be assessed in nonambulatory patients capable of weak dorsiflexion. This could have significance in relation to rehabilitation strategies aimed at improving locomotor performance because there may be prognostic implications for the final gait capacity in individual subjects. From a practical point of view, statistical estimates of short-term synchronization require only short periods of EMG activity in ankle dorsiflexors and therefore are easily translatable to a clinical setting.

\section{G R A N T S}

This work was supported by grants from the Danish National Research Foundation, Elsass Foundation, Bevica Foundation (previously Sahva Foundation), and Canadian Institutes of Health Research to D. Barthélemy.

\section{I S C L OS URES}

No conflicts of interest, financial or otherwise, are declared by the author(s).

\section{REFERENCES}

Alexeeva N, Broton JG, Calancie B. Latency of changes in spinal motoneuron excitability evoked by transcranial magnetic brain stimulation in spinal cord injured individuals. Electroencephalogr Clin Neurophysiol 109: 297303, 1998.

Armstrong DM, Drew T. Forelimb electromyographic responses to motor cortex stimulation during locomotion in the cat. J Physiol 367: 327-351, 1985.

Barthélemy D, Nielsen JB. Corticospinal contribution to arm muscle activity during human walking. J Physiol 588: 967-979, 2010.

Barthélemy D, Willerslev-Olsen M, Lundell H, Knudsen H, BieringSorensen F, Nielsen JB. Impaired transmission in the corticospinal tract and gait disability in persons with partial spinal cord injury. Program No. 469.4. 2008 Neuroscience Meeting Planner. Washington, DC: Society for Neuroscience, 2008. Online.

Bensoussan L, Mesure S, Viton JM, Delarque A. Kinematic and kinetic asymmetries in hemiplegic patients' gait initiation patterns. J Rehabil Med 38: 287-294, 2006.

Bretzner F, Drew T. Contribution of the motor cortex to the structure and the timing of hindlimb locomotion in the cat: a microstimulation study. $J$ Neurophysiol 94: 657-672, 2005.

Brillinger DR. Some aspects of modern population mathematics. Can J Stat 9: 173-194, 1981.

Brouwer B, Bugaresti J, Ashby P. Changes in corticospinal facilitation of lower limb spinal motor neurons after spinal cord lesions. J Neurol Neurosurg Psychiatry 55: 20-24, 1992.

Calancie B, Alexeeva N, Broton JG, Suys S, Hall A, Klose KJ. Distribution and latency of muscle responses to transcranial magnetic stimulation of motor cortex after spinal cord injury in humans. J Neurotrauma 16: 49-67, 1999

Capaday C, Lavoie BA, Barbeau H, Schneider C, Bonnard M. Studies on the corticospinal control of human walking. I. Responses to focal transcranial magnetic stimulation of the motor cortex. J Neurophysiol 81: 129-139, 1999.

Chin R, Hsiao-Wecksler ET, Loth E, Kogler G, Manwaring SD, Tyson SN, Shorter KA, Gilmer JN. A pneumatic power harvesting ankle-foot orthosis to prevent foot-drop (Abstract). J Neuroeng Rehabil 6: 19, 2009.

Courtine G, Roy RR, Raven J, Hodgson J, McKay H, Yang H, Zhong H, Tuszynski MH, Edgerton VR. Performance of locomotion and foot grasping following a unilateral thoracic corticospinal tract lesion in monkeys (Macaca mulatta). Brain 128: 2338-2358, 2005.

Datta AK, Farmer SF, Stephens JA. Central nervous pathways underlying synchronization of human motor unit firing studied during voluntary contractions. J Physiol 432: 401-425, 1991.

Davey NJ, Smith HC, Savic G, Maskill DW, Ellaway PH, Frankel HL. Comparison of input-output patterns in the corticospinal system of normal subjects and incomplete spinal cord injured patients. Exp Brain Res 127: 382-390, 1999.

Drew T. Motor cortical activity during voluntary gait modifications in the cat. I. Cells related to the forelimbs. J Neurophysiol 70: 179-199, 1993.

Drew T, Jiang W, Widajewicz W. Contributions of the motor cortex to the control of the hindlimbs during locomotion in the cat. Brain Res Brain Res Rev 40: 178-191, 2002.

Ellaway PH, Anand P, Bergstrom EM, Catley M, Davey NJ, Frankel HL, Jamous A, Mathias C, Nicotra A, Savic G, Short D, Theodorou S. Towards improved clinical and physiological assessments of recovery in spinal cord injury: a clinical initiative. Spinal Cord 42: 325-337, 2004

Farmer SF, Bremner FD, Halliday DM, Rosenberg JR, Stephens JA. The frequency content of common synaptic inputs to motoneurones studied during voluntary isometric contraction in man. J Physiol 470: 127-155, 1993.

Farmer SF, Halliday DM, Conway BA, Stephens JA, Rosenberg JR. A review of recent applications of cross-correlation methodologies to human motor unit recording. J Neurosci Methods 74: 175-187, 1997.

Halliday DM, Conway BA, Christensen LO, Hansen NL, Petersen NP, Nielsen JB. Functional coupling of motor units is modulated during walking in human subjects. J Neurophysiol 89: 960-968, 2003.

Halliday DM, Rosenberg JR, Amjad AM, Breeze P, Conway BA, Farmer SF. A framework for the analysis of mixed time series/point process data: theory and application to the study of physiological tremor, single motor unit discharges and electromyograms. Prog Biophys Mol Biol 64: 237-278, 1995.

Hansen NL, Conway BA, Halliday DM, Hansen S, Pyndt HS, BieringSorensen F, Nielsen JB. Reduction of common synaptic drive to ankle dorsiflexor motoneurons during walking in patients with spinal cord lesion. J Neurophysiol 94: 934-942, 2005. 
Hansen NL, Hansen S, Christensen LO, Petersen NT, Nielsen JB. Synchronization of lower limb motor unit activity during walking in human subjects. J Neurophysiol 86: 1266-1276, 2001.

Hultborn H, Nielsen JB. Spinal control of locomotion: from cat to man. Acta Physiol (Oxf) 189: 111-121, 2007.

Hummel FC, Cohen LG. Drivers of brain plasticity. Curr Opin Neurol 18: 667-674, 2005.

Jiang W, Drew T. Effects of bilateral lesions of the dorsolateral funiculi and dorsal columns at the level of the low thoracic spinal cord on the control of locomotion in the adult cat. I. Treadmill walking. J Neurophysiol 76: 849-866, 1996.

Kilner JM, Baker SN, Salenius S, Jousmaki V, Hari R, Lemon RN. Task-dependent modulation of $15-30 \mathrm{~Hz}$ coherence between rectified EMGs from human hand and forearm muscles. J Physiol 516: 559-570, 1999.

Lawrence DG, Kuypers HG. The functional organization of the motor system in the monkey. I. The effects of bilateral pyramidal lesions. Brain 91: 1-14, 1968.

Marino RJ, Barros T, Biering-Sorensen F, Burns SP, Donovan WH, Graves DE, Haak M, Hudson LM, Priebe MM. International standards for neurological classification of spinal cord injury. J Spinal Cord Med 26, Suppl. 1: S50-S56, 2003.

McKay WB, Lee DC, Lim HK, Holmes SA, Sherwood AM. Neurophysiological examination of the corticospinal system and voluntary motor control in motor-incomplete human spinal cord injury. Exp Brain Res 163: $379-$ 387, 2005.

Myers LJ, Lowery M, O’Malley M, Vaughan CL, Heneghan C, St Clair GA, Harley YX, Sreenivasan R. Rectification and non-linear pre-processing of EMG signals for cortico-muscular analysis. J Neurosci Methods 124 157-165, 2003.

Nathan PW. Effects on movement of surgical incisions into the human spinal cord. Brain 117: 337-346, 1994.

Nielsen JB. How we walk: central control of muscle activity during human walking. Neuroscientist 9: 195-204, 2003.

Nielsen JB, Brittain JS, Halliday DM, Marchand-Pauvert V, Mazevet D, Conway BA. Reduction of common motoneuronal drive on the affected side during walking in hemiplegic stroke patients. Clin Neurophysiol 119: 2813-2818, 2008.

Norton JA, Gorassini MA. Changes in cortically related intermuscular coherence accompanying improvements in locomotor skills in incomplete spinal cord injury. J Neurophysiol 95: 2580-2589, 2006.

Novak AC, Olney SJ, Bagg S, Brouwer B. Gait changes following botulinum toxin A treatment in stroke. Top Stroke Rehabil 16: 367-376, 2009.

Nudo RJ. Plasticity. NeuroRx 3: 420-427, 2006.

Paoloni M, Mangone M, Scettri P, Procaccianti R, Cometa A, Santilli V. Segmental muscle vibration improves walking in chronic stroke patients with foot drop: a randomized controlled trial. Neurorehabil Neural Repair 23: 819-824, 2009
Perkel DH, Gerstein GL, Moore GP. Neuronal spike trains and stochastic point processes. I. The single spike train. Biophys J 7: 391-418, 1967a.

Perkel DH, Gerstein GL, Moore GP. Neuronal spike trains and stochastic point processes. II. Simultaneous spike trains. Biophys J 7: 419-440, 1967b.

Petersen N, Christensen LO, Morita H, Sinkjaer T, Nielsen J. Evidence that a transcortical pathway contributes to stretch reflexes in the tibialis anterior muscle in man. J Physiol 512: 267-276, 1998a.

Petersen N, Christensen LO, Nielsen J. The effect of transcranial magnetic stimulation on the soleus $\mathrm{H}$ reflex during human walking. J Physiol 513: 599-610, 1998 b.

Petersen NT, Butler JE, Marchand-Pauvert V, Fisher R, Ledebt A, Pyndt HS, Hansen NL, Nielsen JB. Suppression of EMG activity by transcranial magnetic stimulation in human subjects during walking. J Physiol 537: 651-656, 2001.

Petersen NT, Pyndt HS, Nielsen JB. Investigating human motor control by transcranial magnetic stimulation. Exp Brain Res 152: 1-16, 2003.

Plow EB, Carey JR, Nudo RJ, Pascual-Leone A. Invasive cortical stimulation to promote recovery of function after stroke: a critical appraisal. Stroke 40: 1926-1931, 2009.

Ravnborg M. The role of transcranial magnetic stimulation and motor evoked potentials in the investigation of central motor pathways in multiple sclerosis. Dan Med Bull 43: 448-462, 1996.

Rossignol S. Plasticity of connections underlying locomotor recovery after central and/or peripheral lesions in the adult mammals. Philos Trans $R$ Soc Lond B Biol Sci 361: 1647-1671, 2006.

Rothwell JC, Hallett M, Berardelli A, Eisen A, Rossini P, Paulus W. Magnetic stimulation: motor evoked potentials. The International Federation of Clinical Neurophysiology. Electroencephalogr Clin Neurophysiol Suppl 52: 97-103, 1999.

Roy RR, Garfinkel A, Ounjian M, Payne J, Hirahara A, Hsu E, Edgerton VR. Three-dimensional structure of cat tibialis anterior motor units. Muscle Nerve 18: 1187-1195, 1995.

Schubert M, Curt A, Jensen L, Dietz V. Corticospinal input in human gait: modulation of magnetically evoked motor responses. Exp Brain Res 115: 234-246, 1997.

Smits E, Rose PK, Gordon T, Richmond FJ. Organization of single motor units in feline sartorius. J Neurophysiol 72: 1885-1896, 1994.

Thomas SL, Gorassini MA. Increases in corticospinal tract function by treadmill training after incomplete spinal cord injury. J Neurophysiol 94: 2844-2855, 2005.

Weber DJ, Stein RB, Chan KM, Loeb GE, Richmond FJ, Rolf R, James K, Chong SL, Thompson AK, Misiaszek J. Functional electrical stimulation using microstimulators to correct foot drop: a case study. Can J Physiol Pharmacol 82: 784-792, 2004.

Westhout FD, Paré LS, Linskey ME. Central causes of foot drop: rare and underappreciated differential diagnoses. J Spinal Cord Med 30: 62-66, 2007. 